\title{
A new method for in vitro feeding of Rhipicephalus australis (formerly Rhipicephalus microplus) larvae: a valuable tool for tick vaccine development
}

\author{
Jos J. A. Trentelman ${ }^{1 *}$, Jos A. G. M. Kleuskens ${ }^{1}$, Jos van de Crommert $^{2}$ and Theo P. M. Schetters ${ }^{3,4}$
}

\begin{abstract}
Background: Rhipicephalus microplus is a hard tick that has a major impact on cattle health in tropical and subtropical regions because it feeds on cattle and is implicated in the transmission of pathogens that cause diseases such as bovine anaplasmosis and babesiosis. Presently, acaricides are used to control tick infestation but this is becoming increasingly less effective due to the emergence of tick strains that are resistant to one or more classes of acaricides. Anti-tick vaccines are a promising alternative to control tick infestation in cattle. The life-cycle and host preference of $R$. microplus, however, makes vaccine research in cattle costly and would therefore greatly benefit from an in vitro screening system.
\end{abstract}

Methods: To this aim, a stacked 24-well in vitro feeding system was designed in which the blood meal was administered in a chamber on top of the compartment containing the ticks, exploiting their anti-gravitational tendency. Both compartments were separated by a special feeding membrane, which was made by applying a silicone mixture to a gold beater's skin (baudruche membrane) with a paint roller to create a slightly uneven surface of 17-40 $\mu \mathrm{m}$ variable thickness. To further stimulate feeding, the membrane was treated with bovine hair extract and the unit was placed at $37^{\circ} \mathrm{C}$ with $90 \% \mathrm{RH}$ and $5 \% \mathrm{CO}_{2}$.

Results: Using this set-up with Rhipicephalus australis (formerly Rhipicephalus microplus), a larval engorgement rate of up to $71 \%$ could be achieved. The larvae could successfully feed on blood, but also on serum. The latter allows easy screening of the effect of sera that are raised against tick proteins on feeding. As an example, serum from cattle that were vaccinated with the Bm86 midgut protein of $R$. microplus significantly reduced larval engorgement rates by $42 \%$.

Conclusion: The in vitro feeding system's high throughput design and its ability to measure statistically significant anti-tick effects in sera from immunized cattle enables screening of multiple vaccine candidates in a cost-effective manner.

Keywords: Artificial tick feeding, In vitro screening, Rhipicephalus australis, Rhipicephalus microplus, Larvae

\footnotetext{
*Correspondence: j.j.trentelman@amc.uva.nl

${ }^{1}$ Center for Experimental and Molecular Medicine, Academic Medical Center

Amsterdam, meibergdreef 9, 1105, AZ, Amsterdam, Netherlands

Full list of author information is available at the end of the article
} 


\section{Background}

Rhipicephalus microplus is a hard tick that has a major impact on cattle health in tropical and subtropical regions. This tick species can transmit a range of diseases, including bovine anaplasmosis and babesiosis. Moreover, ticks can also adversely affect cattle production directly by feeding alone [1]. It is therefore of great importance to control tick infestations to ensure livestock health and productivity. To date, tick control heavily depends on the use of tick-resistant breeds and treatment of susceptible breeds with acaricides, but tick resistance to these acaricides is becoming problematic [2].

Anti-tick vaccines are potential alternatives for acaricides to control tick infestation. Anti-tick immunity is known to consist of both cellular and humoral factors [3-6]. Transfer of tick immune serum to naïve animals resulted in tick rejection indicating an important role for antibodies [7, 8]. Early studies have shown that vaccination with crude tick antigen preparations induced antibodies that interfered with feeding and subsequent further development $[9,10]$. With the advent of recombinant protein techniques, single protein antigens could be evaluated for protective activity. This led to the discovery of Bm86, a tick midgut antigen first described in 1989 [11]. This antigen is the basis of two commercial anti-tick vaccines $\left(\right.$ Gavac $^{\mathrm{Tm}}$, Heber biotec ${ }^{\mathrm{Tm}}$; TickGard, Merck Animal Health) [12, 13]. In experimental studies, Bm86 vaccination reduces tick numbers up to $74 \%$ and reduces tick fertility, combining the overall efficacy of up to $91 \%$ depending on the $R$. microplus challenge strain [14]. Protection by $\mathrm{Bm} 86$ vaccination is $\operatorname{IgG}$ mediated and protection can be correlated to IgG titers $[15,16]$. Although Bm86 vaccination reduces the number of acaracide treatments in the field, a lack of a knock-down effect hampers its wide spread use [12, 17, 18]. More research is needed to improve anti-tick vaccines by additional and/or more efficacious antigens.

Currently, vaccination-challenge trials in cattle are being used to evaluate and select $R$. microplus vaccine candidate antigens. Aside from the fact that this restrains the number of experiments that can be done from an animal welfare point of view, it is also very costly and time consuming. As antibodies are an essential mediator in induced and natural anti-tick immunity, an in vitro feeding model of $R$. microplus is an attractive alternative to evaluate the inhibitory effect of such sera on engorgement and further tick development. Different techniques have been tried with varying degrees of success. These techniques can be divided in two approaches: tube feeding and membrane feeding. In vitro feeding of blood with tubes has proven to be a successful method for feeding $R$. microplus in vitro; ticks are forced to feed by placing a glass tube with blood over the hypostome or the entire mouthparts [19-21]. A major drawback of tube feeding is that only semi-engorged adult females can be used; they are eager to imbibe blood and they have the larger feeding apparatus needed for this technique. Larvae and nymphs cannot be fed using capillaries.

Membrane feeding, which more closely mimics the natural feeding process, has been successfully developed for several tick species [22-25]. This technique is not limited to adult ticks, but can also be used to feed larval hard ticks in vitro; either with a completely artificial feeding membrane [23, 24] or derived from animal skin $[25,26]$. These studies also showed that the blood meal could be presented below or above the tick compartment. However, in the few described cases of membrane feeding of $R$. microplus larvae, embryonated hen egg membranes or bovine skin slices were used and the ticks were placed on top of the blood meal [27-29]. For adult $R$. microplus ticks, these membranes were subsequently replaced by artificial feeding membranes prepared from gut intestines (baudruche membrane) [30]. The baudruche membrane was coated with layers of contact cement and treated with bovine skin extract to stimulate the feeding process. These attempts met with limited success, and presently there is still no recorded in vitro membrane feeding system that allows reproducible feeding of $R$. microplus larvae. Only recently ribosomal DNA and morphological analysis resulted in the reinstatement of $R$. australis as a separate species from $R$. microplus [31]. Morphologically there are only small differences between $R$. microplus and $R$. australis larvae, the hypostome length is however identical, making $R$. australis an excellent model for both species. Here we describe the development of an in vitro membrane feeding system for $R$. australis larvae that can be used to evaluate the inhibitory activity of antisera against tick antigens. The system described here enables high-throughput screening for tick vaccine candidate antigens.

\section{Methods}

Tick larvae

Tick larvae were obtained from a colony of $R$. australis (formerly $R$. microplus) originating in Australia that was routinely passaged on Holstein calves (Merck Animal Health Innovation $\mathrm{GmbH}$, Schwabenheim, Germany). Fully engorged female ticks were collected from the calves and allowed to oviposit in petri dishes. The resulting egg masses were collected in laboratory tubes and allowed to hatch at $22{ }^{\circ} \mathrm{C}$ and $90 \%$ humidity. Four to 6week-old $R$. australis larvae were used in the feeding experiments.

\section{Preparation of cow hair extract}

Hair was collected from the flank of tick naïve Friesian Holstein cattle. Fifty gram of hair was incubated for $20 \mathrm{~min}$ at room temperature with $250 \mathrm{ml}$ dichloromethane 
(Merck, Darmstadt, Germany), supernatant was collected. This was repeated twice with $100 \mathrm{ml}$ dichloromethane. The collected dichloromethane extracts were pooled and centrifuged at $3000 \times \mathrm{rpm}$ for $20 \mathrm{~min}$. Supernatant was collected and concentrated to a low volatile lipid content of $7 \mathrm{mg} / \mathrm{ml}$ by heating at $40{ }^{\circ} \mathrm{C}$.

\section{Feeding unit design}

Feeding units were designed using three $30 \mathrm{~mm}$ Perspex plates that were perforated to create a 24-well culture plate design (total size $150 \mathrm{~mm}$ length $\times 110 \mathrm{~mm}$ width, wells are $15.5 \mathrm{~mm}$ in diameter placed within $127 \mathrm{~mm} \times$ $85 \mathrm{~mm}$ ). The plates were assembled with 8 steel bolts to obtain a three-layered stack (Fig. 1). The wells of the upper layer contained the test serum or blood (Fig. 1a, layer 1). The top was covered with an Elisa plate seal or Parafilm to maintain sterility. This layer was separated from the second layer by the feeding membrane that was placed with the silicone side facing downwards (Fig. 1a, layer 2). The wells of the second layer contained the ticks (Fig. 1a, layer 3). To contain the ticks, this layer was separated from the third layer with netting (Fig. 1a, layer 4). The third layer functioned as support and allowed gas exchange between the environment and the tick compartments (Fig. 1a, layer 5).

\section{Feeding membrane}

The basis of the feeding membranes was goldbeater's skin of less than $30 \mu \mathrm{m}$ thickness (Preservation Equipment Ltd, Norfolk, UK). Goldbeater's skin is comparable to a baudruche membrane; both are made from bovine intestine. The membranes were treated with silicon to add strength and flexibility. Silicone mixture was prepared: 15 g Wacker silicone E4, 9 gr Silicone AP 200 (Sigma-Aldrich, St. louis, USA) and $5.8 \mathrm{~g}$ Hexane (Sigma-Aldrich). After carefully mixing, $1.5 \mathrm{mg}$ silicone mixture per $\mathrm{cm}^{2}$ was applied with either a rubber sheet or a gloss paint roller. The siliconized membrane was left to polymerize overnight at room temperature and ambient humidity. Final membrane thickness was measured with a micrometer. Membranes with a maximal thickness of $40 \mu \mathrm{m}$ were used for feeding.

\section{Feeding unit assembly}

Figure 1a shows a schematic view of the feeding unit. As a first step, only the first two Perspex plates (Fig. 1a, layer 1 and 3) were assembled together with the siliconized feeding membrane (Fig. 1a, layer 2) in between, and placed upside down. Subsequently, $75 \mu \mathrm{l}$ dichloromethane bovine hair extract was added to each well and left to dry for $30 \mathrm{~min}$ at room temperature to apply bovine scent to the siliconized side of the feeding membrane. Next, $R$. australis larvae were added to the wells (Fig. 1a, layer 3) (approximately 100 larvae per well). A piece of net curtain was used to cover the plate and contain the larvae, after which the lower plate was immediately mounted using the bolts (Fig. 1a, layer 4 and 5). The unit was then put upright. This system design stimulates contact between the blood (or serum) (Fig. 1a, layer 1) and the larvae; red blood cells settle on top of the feeding membrane, and

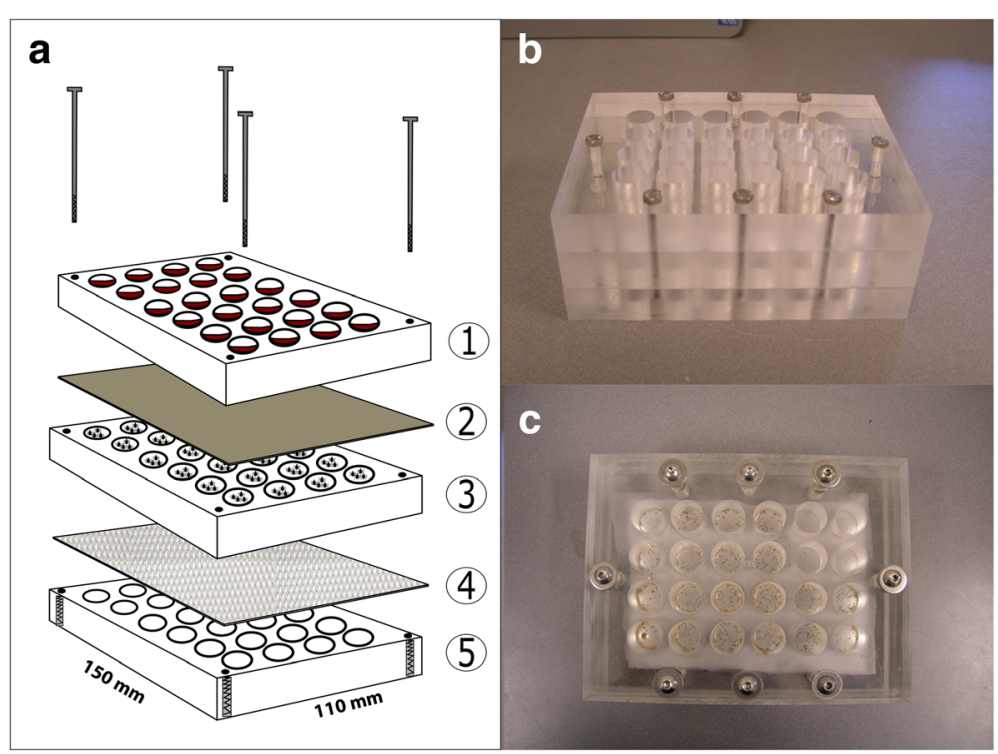

Fig. 1 In vitro feeding unit. a Schematic representation: The wells of the upper layer contained the test serum or blood (1). The top was covered with an Elisa plate seal or Parafilm to maintain sterility. This layer was separated from the second layer by the feeding membrane that was placed with the silicone side facing downwards (2). The wells of the second layer contained the ticks (3). To contain the ticks, this layer was separated from the third layer with netting (4). The third layer functioned as support and allowed gas exchange between the environment and the tick compartments (5). b A frontal view of the unit. c Top view of the feeding unit, R. australis larvae can be seen through the membrane 
larvae due to their natural anti-gravitational tendency crawl up to the underside of the membrane.

\section{Feeding unit operation}

The wells of the upper plate with the baudruche side of the feeding membrane at the bottom were disinfected using $70 \%$ ethanol and left to dry. Test samples (blood or serum) were pre-warmed at $37{ }^{\circ} \mathrm{C}$ and subsequently added to the wells of the upper plate. The upper plate was sealed with an ELISA plate cover or Parafilm. The unit was placed in a dark $\mathrm{CO}_{2}$ incubator at $37{ }^{\circ} \mathrm{C}, 90 \%$ $\mathrm{RH}$ and $5 \% \mathrm{CO}_{2}$ (as a feeding stimulus) for up to $72 \mathrm{~h}$ to allow larvae to feed. Feeding was stopped by placing the feeding unit overnight at $-20{ }^{\circ} \mathrm{C}$ thus freezing the larvae. The percentage of larvae that were engorged was determined using a stereomicroscope. Larvae were scored to be engorged when having an enlarged abdomen of at least 2 times the dorsal shield (Fig. 2). To secure objectivity and prevent subconscious skewing, details about the test materials were kept secret to the evaluator (see below; Statistical evaluation).

\section{Bovine blood}

Blood was collected from healthy tick-naive Friesian Holstein cattle in $500 \mathrm{ml}$ glass bottles under vacuum with sterilized glass beads. The blood was defribinated by gently shaking. The defibrinated blood was supplemented with glucose (Sigma-Aldrich) to a concentration of $3 \mathrm{~g} / \mathrm{l}$ and stored at $4{ }^{\circ} \mathrm{C}$. Before adding the blood to the feeding well, $5 \mu$ l gentamycin $(10 \mathrm{mg} / \mathrm{ml}$; Sigma-Aldrich) and $100 \mu \mathrm{l}$ sterile filtered ATP $(0.1 \mathrm{M}$ in $0.9 \% \mathrm{NaCl}$; Sigma-Aldrich) was added to every $10 \mathrm{ml}$ of blood. Six hundred microliters of bovine blood was added to each well and changed twice daily.

\section{Bovine serum}

To produce normal serum, blood from healthy ticknaive Holstein Friesian cattle was collected in BD Vacutainer ${ }^{\ominus}$ Plus plastic serum tubes (BD, New Jersey, USA). Blood was allowed to clot for $1 \mathrm{~h}$ at $37^{\circ} \mathrm{C}$, centrifuged for $15 \mathrm{~min}$ at $1000 \mathrm{RCF}$, serum was harvested and stored at $-20{ }^{\circ} \mathrm{C}$.

\section{Anti Bm86 bovine serum}

Five healthy, tick-naive Friesian Holstein cattle were vaccinated twice at a 3-week interval with Bm86 produced in the Baculovirus expression system in water in oil adjuvant (Montanide ISA 50 V2, Seppic, Paris, France). Two weeks after the last vaccination, blood was collected for serum production. Serum was pooled before feeding.

Before serum was added to the in vitro feeding system, each $10 \mathrm{ml}$ of serum was supplemented with $5 \mu \mathrm{l}$ gentamycin (10 mg/ml; Sigma-Aldrich). Six hundred microliters of serum was added to each well and changed twice daily.

\section{Bm86 ELISA}

Anti Bm86 bovine serum titers were determined in a sandwich ELISA. In short, rabbit anti-Pichia expressed Bm86 (5 $\mu \mathrm{g} / \mathrm{ml}$ in CBB buffer) was coated overnight on a Greiner F ELISA plate at room temperature. The wells were subsequently blocked for $1 \mathrm{~h}$ with $200 \mu \mathrm{l} /$ well $1 \% \mathrm{w} / \mathrm{v}$ BSA in $0.04 \mathrm{M}$ isotonic PBS at $37{ }^{\circ} \mathrm{C}$. Next, $100 \mu \mathrm{l} /$ well Baculovirus expressed Bm86 was added to the plate $(0.12 \mu \mathrm{g} / \mathrm{ml}$ in $1 \% \mathrm{w} / \mathrm{v}$ BSA in EIA-tween 80 buffer) and left to incubate for $2 \mathrm{~h}$ at $37{ }^{\circ} \mathrm{C}$. Serum was diluted (in $1 \% \mathrm{w} / \mathrm{v}$ BSA in GLD1 buffer supplemented with $10 \% \mathrm{v} / \mathrm{v}$ normal dog serum) and $100 \mu \mathrm{l} /$ well subsequently added to the plate for $1 \mathrm{~h}$ incubation at $37^{\circ} \mathrm{C}$. Goat anti-bovine IgG-HRP (Jackson ImmunoResearch Inc., West Grove, PA, USA,) was 2500 times diluted in $1 \% \mathrm{w} / \mathrm{v}$ BSA in EIA-tween 80 buffer and $100 \mu \mathrm{l} /$ well added to incubate for $1 \mathrm{~h}$ at $37{ }^{\circ} \mathrm{C}$. Finally, $100 \mu \mathrm{l} /$ well substrate $(185 \mu \mathrm{l}$ TMB and $1 \mathrm{ml}$ UP-buffer in $10 \mathrm{ml}$ water for injection) was added and left to incubate for $15 \mathrm{~min}$ in the dark at room temperature. The reaction was stopped with $50 \mu \mathrm{l} /$ well $4 \mathrm{~N} \mathrm{H}_{2} \mathrm{SO}_{4}$ and OD was measured at $450 \mathrm{~nm}$.

\section{Statistical evaluation}

Samples were tested at least as quadruplicates. To prevent plate-position effects on feeding, samples were

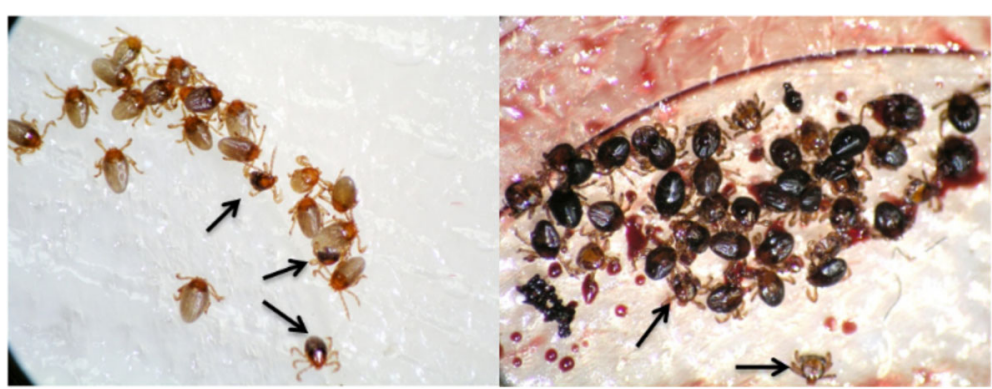

Fig. 2 R. australis larvae on the feeding membrane. Most of the larvae have engorged with serum (left) or blood (right). Examples of larvae considered unengorged are indicated with an arrow 
allocated to the feeding unit such that they were evenly distributed over the plate. The code was kept secret to the evaluator until after determination of the engorgement rate in each well. From the individual values the average engorgement rate was calculated. The obtained results were statistically evaluated using Graphpad Prism (Graphpad Prism 5, Graphpad software Inc.).

\section{Results}

The goal of our study was to develop a feeding system that facilitates in vitro feeding of R. microplus in a high throughput mode. For this purpose, a 24-well format tick feeding unit consisting of Perspex plates was designed in which the feeding membrane and blood/serum are placed above the larvae, stimulating tick attachment and feeding due to the negative geotaxis of the larvae (Fig. 1). The amount of feeding medium required was $600 \mu \mathrm{l} /$ well, which is quite low and very suitable for screening purposes.

\section{Membrane preparation}

Because larvae of both $R$. australis and $R$. microplus have very short mouth parts (around $70 \mu \mathrm{m}$ ) we selected goldbeater's skin with a thickness less than $30 \mu \mathrm{m}$ as a base. To add strength and flexibility, silicone was applied with a rubber sheet as described by Kröber \& Guerin [22]. Initial results with this method revealed an engorgement rate of around $10 \%$ after $48 \mathrm{~h}$ of feeding on bovine blood (data not shown). Because the preparation of feeding membranes with a rubber sheet is somewhat cumbersome, we compared application of silicon by using a rubber sheet or by using a gloss paint roller. For each application method, eight wells were used. Results showed that the engorgement rate after feeding for $48 \mathrm{~h}$ on bovine blood was statistically significant higher using membranes prepared with the paint roller $(29.4 \%$ average engorgement) as compared to membranes prepared with the rubber sheet $(13.6 \%$ average engorgement; $\left(t_{(14)}=4.363, P=0.0006\right.$ Fig. 3$)$. Using the paintroller to apply the silicone mixture, it became possible to prepare membranes of $17-40 \mu \mathrm{m}$ thickness, depending on the thickness of the used goldbeater's skin. Microscopic examination of the membranes produced with the paint roller revealed an irregular relief.

\section{Optimization feeding time on bovine blood}

As it was not known whether larvae that did not engorge after $48 \mathrm{~h}$ of feeding were incapable of feeding or would do so when given more time, we determined the engorgement rate at different time periods after initiation of feeding. Three feeding units were used to determine the engorgement rate at 24, 48 and $72 \mathrm{~h}$ after the start of the experiment. At each time point one feeding unit was frozen for evaluation. Results showed that the

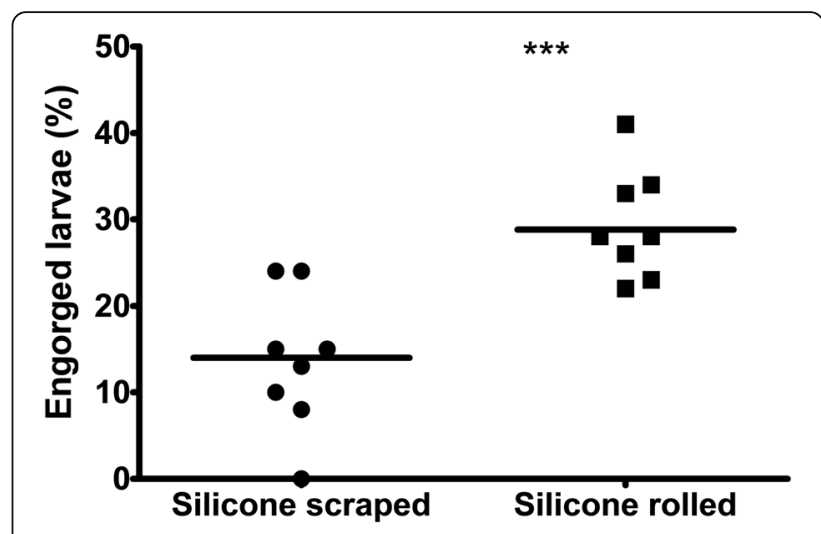

Fig. 3 Effect of silicone application method. Percentage of ticks considered to have engorged after $48 \mathrm{~h}$. Silicone application with a paint roller double the number of larvae that have engorged

average engorgement rate of larvae that were fed bovine blood increased from $12 \%$ after $24 \mathrm{~h}$ to $54 \%$ after $48 \mathrm{~h}$ (Fig. 4). Adding another $24 \mathrm{~h}$ to the feeding time did not increase the average engorgement rate further.

\section{Feeding on bovine serum}

With the basic requirements for successful feeding of larvae on blood set, we determined whether larvae would also feed on serum only. This would be of prime importance for screening tick vaccine candidates using serum of immunized calves. To also test variation due to different batches of siliconized membranes, two different units were set up with membranes from different batches. In each of the units, part of the wells was filled with bovine blood and another part with bovine serum. The larvae were allowed to feed for $48 \mathrm{~h}$ on these substrates after which the engorgement rate was determined.

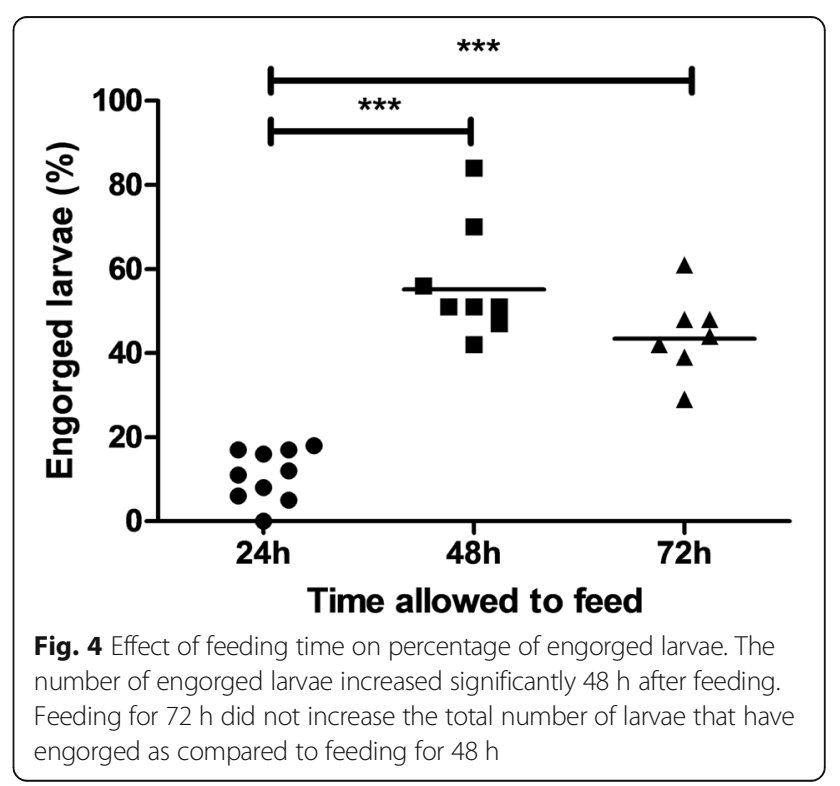


Results showed that the engorgement rate of larvae feeding on bovine serum was somewhat lower (59\%) than that observed when larvae were fed on bovine blood (71\%; Fig. 5). This difference was statistically significant $\left(t_{(14)}=3.858, P=0.0017\right)$. There was no apparent difference between the engorgement rates obtained with the two different batches of siliconized membranes.

\section{Effect of anti Bm86 bovine serum}

To validate the use of the feeding system for the selection of putative tick vaccine candidates we used Bm86 midgut antigen as an example. Serum was prepared from calves prior to and after immunization with recombinant Bm86 produced in the Baculovirus expression system. An ELISA capturing recombinant Baculovirus expressed Bm86 with rabbit anti-Bm86 IgG against Pichia expressed Bm86 was used to measure the antibody titer of the anti-Bm86 calf serum. Results showed that vaccination with $\mathrm{Bm} 86$ resulted in a $2 \log$ antibody end titer of 17 (cut off calculation: Bmin*2) (Fig. 6). Next, both sera were used to feed larvae in vitro to determine the effect of anti Bm86 antibodies on engorgement. Each serum was tested in eight-fold, larvae were allowed to feed for $48 \mathrm{~h}$. Results showed that the engorgement rate of larvae that were fed on anti-Bm86 serum was reduced by $42 \%$ as compared to controls (Fig. 7). This result was statistically significant $\left(t_{(14)}=3.497, P=0.0036\right)$.

\section{Discussion}

The search for effective anti-tick vaccines is currently restricted by the use of calves to determine the efficacy of

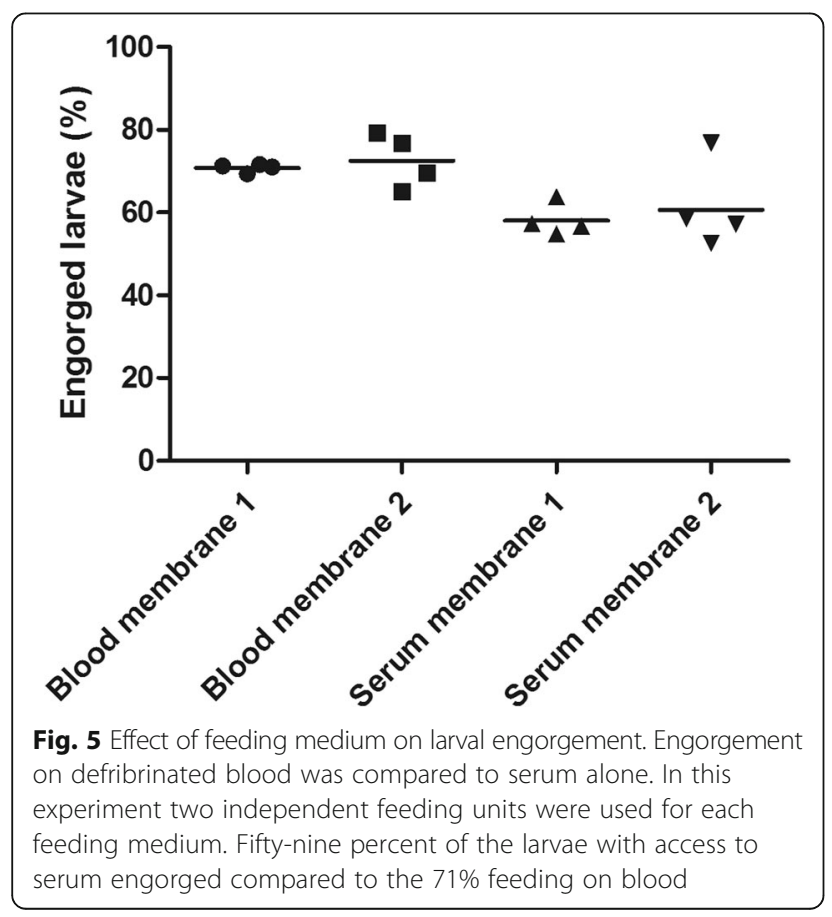

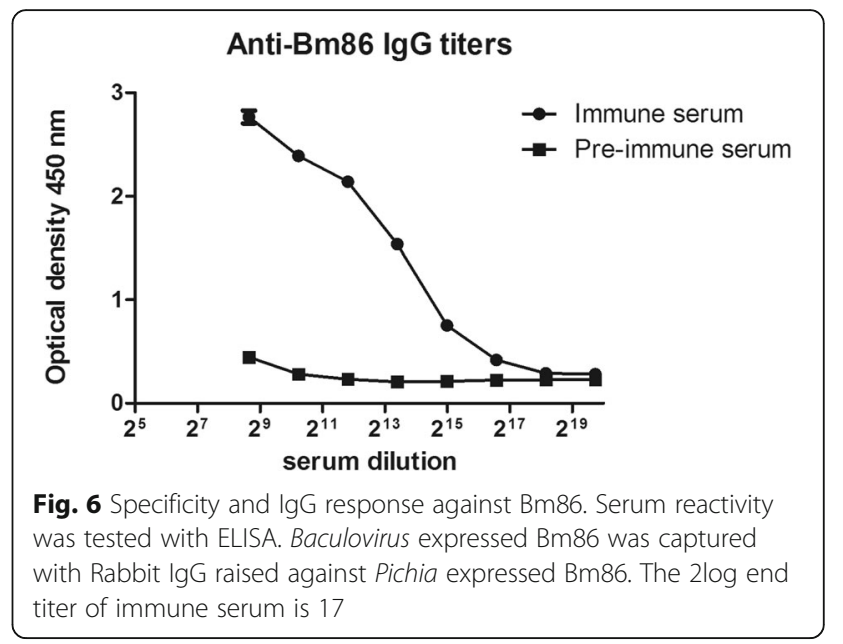

immunization in vaccination-challenge experiments. An in vitro tick feeding system that allows determining the effect of sera from vaccinated calves on tick viability would be advantageous. In vitro feeding systems using artificial feeding membranes have been developed for several different tick species, but until now no such system has been developed for $R$. microplus larvae. This has been hypothesized to be due to the fact that $R$. microplus have a very short hypostome (approximately $70 \mu \mathrm{m}$ ) $[23,31,32]$, which is too short to penetrate the type of artificial membranes that are used for feeding of ticks in vitro [23]. Here we show that a feeding membrane based on goldbeater's skin that is coated with silicon effectively supports feeding of $R$. australis (formerly $R$. microplus) larvae in vitro. $R$. australis ticks were considered to be $R$. microplus until comprehensive morphological and genetic analysis showed that this group of ticks should be divided into two species [31]. However, since the larvae from both species have the same hypostome length and both species are affected by Bm86 vaccination, the results presented here with $R$.

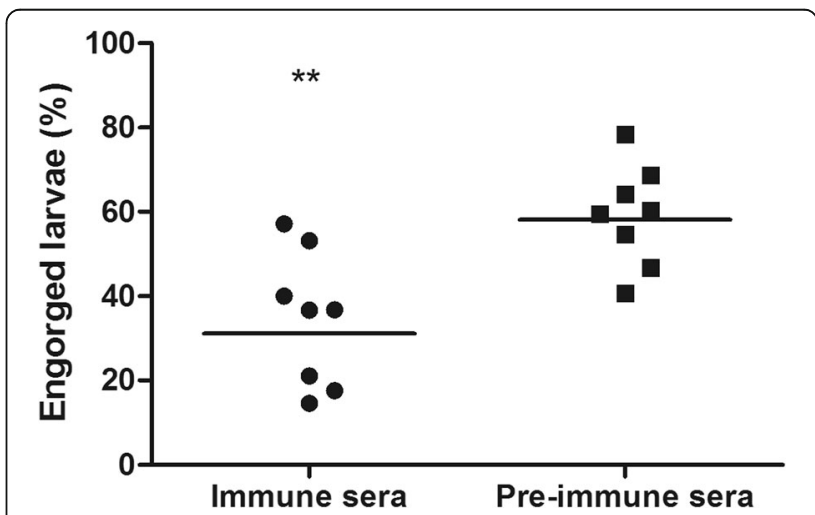

Fig. 7 Effect of Bm86 vaccination on percentage of larvae that have engorged. Vaccination reduced the number of engorged larvae by $42 \%$ 
australis can be easily translated to $R$. microplus larvae [31]. In the current in vitro assay, the method used to coat the goldbeater's skin with silicon appeared to be an important variable; application of silicon with a gloss paint roller produced better membranes as compared to application by wiping with a rubber sheet. It is suggested here that the irregular surface texture produced with the paint roller is crucial, probably because it provides support for the larvae to attach to the membrane and the fact that the membrane is at some points thinner than at other points allows successful penetration by the hypostome. Importantly, between membranes there was little variation in larval feeding, illustrating the reproducibility of the membrane production. The engorgement rate of the larvae was determined by estimating their size visually, using a stereomicroscope. It could be argued that measuring larval weight after feeding, instead of size, would give a more objective measurement of engorgement. Unfortunately, the attachment to the membrane at the moment of scoring and the number of larvae used (approx. 2400 in one in vitro feeding unit) make it technically nearly impossible to remove all larvae undamaged for accurate weighing.

The results presented here show that there is considerable variation in the engorgement rate per well. Because of this variation it is important to have at least quadruplicate measurements to calculate reliable average engorgement rates. The 24-well plate set-up presented here, enables testing different feeding substrates in a single feeding unit. Additional advantages of the system are that the feeding substrates can be presented to the ticks aseptically, relatively small volumes of blood/serum are required, the feeding membrane is located at the top of the wells that contain the larvae (larvae tend to crawl upward), and relatively large numbers of larvae can be used per well, which adds to the accuracy of measuring engorgement rates.

Results showed that larvae achieved the maximum engorgement rate after $48 \mathrm{~h}$ of feeding; adding another $24 \mathrm{~h}$ did not increase the engorgement rate further. Hence, assessing the inhibitory effect of anti-tick compounds (be it acaricides or sera from immunized animals) can be completed in two days in vitro if the targeted antigen is expressed in the first $48 \mathrm{~h}$ after tick attachment. For antigens expressed at later time points longer feeding time might be required. This is however a major advantage over in vivo vaccination-challenge experiments in calves that are currently used to determine the effect of vaccination on tick infestation. Such experiments take on average 3 to 4 weeks before larvae have developed to fully engorged female ticks. Clearly, in vivo challenge with larvae does not only estimate the effect of treatment on larvae but also on nymphs and adult ticks, because $R$. microplus and $R$. australis are one-host ticks with most of the larvae developing to fully engorged females on the same host. The system presented here is validated to test the effects of anti-tick compounds on larvae. Although the system enables the development of larvae to nymphs (up to $11 \%$ of applied larvae moulted into nymphs after two-week incubation in a $\mathrm{CO}_{2}$ incubator at $37{ }^{\circ} \mathrm{C}, 90 \% \mathrm{RH}$ and $5 \% \mathrm{CO}_{2}$ with only an initial $600 \mu \mathrm{l}$ tick naive bovine serum added to the feeding unit; data not shown), it remains to be investigated whether it can also be used to feed nymphs. It is not likely that it can be used to feed adults because these are too large for a 24-well format. Alternatively, a 6-well format could be developed for mature stages.

We observed variation in the engorgement rate among different experiments using the same substrates for feeding. Given the fact that the membranes could be prepared in a reproducible manner, the most likely explanation for this variation is due to differences in the viability of the larvae that were used. Consequently, it is advised to test the effect of anti-tick compounds in the feeding substrate using larvae of similar age derived from a single batch.

Although it has been shown before that larvae feed on tissue fluids containing very few erythrocytes [33, 34], it remained to be determined whether serum alone would suffice, especially if the system is to be used to determine the effect of anti-tick antibodies on larval development. Kemp et al. have shown in 1975 that R. microplus larvae indeed feed on medium enriched with bovine serum proteins [28]. In addition, it has been shown that serum as a blood meal does not repel larval attachment, however it is not clear if the larvae feed on serum alone [27]. Using the in vitro system presented here, larvae could feed on serum alone, although engorgement rates were somewhat reduced (59\% fed on serum as compared to $71 \%$ on defibrinated blood). Hence, the system appears suited to aid in the selection of tick vaccine candidate antigens by feeding serum from immunized animals and comparing the engorgement rates with that obtained with normal serum controls.

For the present investigation, Bm86 was chosen as a reference antigen. Pooled serum from calves that were vaccinated with recombinant $\mathrm{Bm} 86$ reduced engorgement of larvae by $42 \%$. This indicates that the Bm86 antigen is indeed expressed in the larval stage of $R$. microplus as has been found previously by qPCR [35], and more importantly is exposed to ingested antibodies directed against the antigen. Since $R$. microplus is a onehost tick, one would expect that antibodies against Bm86 would also affect nymphs next to larvae and adults. The reduction in engorgement by $\mathrm{Bm} 86$ immune sera in vitro is difficult to compare to earlier published in vivo results. Aside from differences in antigens, adjuvants and vaccine formulations used to vaccinate the cattle, field circumstances such as cattle breed, presence 
of different tick strains and epidemiological situations all influence efficacy of vaccination.

\section{Conclusion}

A 24 well in vitro feeding system has been developed to successfully feed Rhipicephalus australis larvae in vitro. By applying a silicone mixture with a paint roller on a baudruche sheet, membranes $<40 \mu \mathrm{m}$ thick were made that can facilitate the short hypostome of $R$. australis and $R$. microplus larvae. Using these membranes in the 24-well in vitro system, larvae were able and willing to feed on defibrinated blood as well as serum alone. Feeding larvae on sera raised against the reference antigen Bm86 reduced tick engorgement with $42 \%$, thereby confirming the feeding systems ability to discriminate anti-tick effects in vitro.

Importantly, it is envisaged that the feeding system can be very useful to determine the potential protective effect of vaccines that contain a mixture of one or more tick antigens. Instead of vaccinating cattle with a plethora of different vaccine formulations, the system allows testing the effect of combinations of anti-tick antibodies by mixing different mono-specific antisera directed against individual tick antigens.

In summary, the in vitro feeding system presented here allows reproducible feeding of $R$. australis larvae on bovine blood and serum. The system can be used for screening tick vaccine candidate antigens and as such accelerate the development of improved anti-tick vaccines.

\section{Acknowledgments}

We like to thank Dr. Heike Williams, Dr. Hartmut Zoller, Mirjam Békefi and Bernd Jürgens from MSD Animal Health Innovation GmbH, Research Antiparasitics for their support and the supply of ticks.

\section{Funding}

The research was funded by MSD Animal Health. Aside from the authors, MSD Animal Health had no role in the study design, data collection and analysis or preparation of the manuscript.

\section{Availability of data and materials}

The datasets supporting the conclusions of this article are included within the article.

\section{Authors' contributions}

TS initiated and supervised the study. JT wrote the manuscript and performed membrane construction and feeding experiments. JK, JC and JT participated in the cattle vaccinations, blood collection and ELISA's. All authors contributed to the design of the in vitro feeding unit. All authors read and approved the final manuscript.

\section{Competing interests}

All authors were employed by MSD Animal Health at the time of the described research.

\section{Consent for publication \\ Not applicable.}

\section{Ethics approval}

Cattle vaccinations were approved by the animal ethical committee of MSD Animal Health.

\section{Publisher's Note}

Springer Nature remains neutral with regard to jurisdictional claims in published maps and institutional affiliations.

\section{Author details}

${ }^{1}$ Center for Experimental and Molecular Medicine, Academic Medical Center Amsterdam, meibergdreef 9, 1105, AZ, Amsterdam, Netherlands. ${ }^{2}$ Aduro Biotech Europe, Kloosterstraat 9, 1101, RX, Boxmeer, Netherlands. ${ }^{3}$ ProtActivity R\&D, Sering 36, 5432, DD, Cuijk, Netherlands. ${ }^{4}$ ClinVet International, Uitzich Road, Bainsvlei, 9338 Bloemfontein, South Africa.

Received: 11 July 2016 Accepted: 7 March 2017

Published online: 23 March 2017

\section{References}

1. Jonsson NN. The productivity effects of cattle tick (Boophilus microplus) infestation on cattle, with particular reference to Bos indicus cattle and their crosses. Vet Parasitol. 2006;137(1-2):1-10.

2. George JE, Pound JM, Davey RB. Chemical control of ticks on cattle and the resistance of these parasites to acaricides. Parasitol. 2004;129(SupplementS1): S353-66.

3. Brossard M, Wikel SK. Immunology of interactions between ticks and hosts. Med Vet Entomol. 1997;11(3):270-6.

4. Brossard M, Wikel SK. Tick immunobiology. Parasitol. 2004;129:S161-76.

5. Wikel SK. Host immunity to ticks. Annu Rev Entomol. 1996;41(1):1-22.

6. Labuda M, Trimnell AR, Lickova M, Kazimirova M, Davies GM, Lissina O, et al. An antivector vaccine protects against a lethal vector-borne pathogen. PLoS Pathog. 2006;2(4):e27.

7. Brossard M, Girardin P. Passive transfer of resistance in rabbits infested with adult Ixodes ricinus L: humoral factors influence feeding and egg laying. Experientia. 1979;35(10):1395-7.

8. Roberts JA, Kerr JD. Boophilus microplus: passive transfer of resistance in cattle. J Parasitol. 1976;62(3):485-8.

9. Allen JR, Humphreys SJ. Immunisation of guinea pigs and cattle against ticks. Nature. 1979;280(5722):491-3.

10. Brown SJ, Shapiro SZ, Askenase PW. Characterization of tick antigens inducing host immune resistance. I. Immunization of guinea pigs with Amblyomma americanum-derived salivary gland extracts and identification of an important salivary gland protein antigen with guinea pig anti-tick antibodies. J Immunol. 1984;133(6):3319-25.

11. Willadsen P, Riding GA, McKenna RV, Kemp DH, Tellam RL, Nielsen JN, et al. Immunologic control of a parasitic arthropod. Identification of a protective antigen from Boophilus microplus. J Immunol. 1989;143(4):1346-51.

12. Jdl F, Almazán C, Canales M, Pérez de la Lastra JM, Kocan KM, Willadsen P. A ten-year review of commercial vaccine performance for control of tick infestations on cattle. Anim Health Res Rev. 2007;8(01):23-8.

13. Jonsson NN, Matschoss AL, Pepper P, Green PE, Albrecht MS. Hungerford Jet al. Evaluation of TickGARDPLUS, a novel vaccine against Boophilus microplus, in lactating Holstein-Friesian cows. Vet Parasitol. 2000;88(3-4):275-85.

14. de la Fuente J, Rodriguez M, Montero C, Redondo M, Garcia-Garcia JC, Mendez L, et al. Vaccination against ticks (Boophilus spp.): the experience with the Bm86-based vaccine Gavac. Genet Anal. 1999;15(3-5):143-8.

15. de la Fuente J, Rodriguez M, Redondo M, Montero C, Garcia-Garcia JC, Mendez $L$, et al. Field studies and cost-effectiveness analysis of vaccination with Gavac against the cattle tick Boophilus microplus. Vaccine. 1998;16(4):366-73.

16. Kemp DH, Pearson RD, Gough JM, Willadsen P. Vaccination against Boophilus microplus: localization of antigens on tick gut cells and their interaction with the host immune system. Exp Appl Acarol. 1989;7(1):43-58.

17. Suarez M, Rubi J, Pérez D, Cordova V, Salazar Y, Vielma A, et al. High impact and effectiveness of Gavac $^{\text {TM }}$ vaccine in the national program for control of bovine ticks Rhipicephalus microplus in Venezuela. Livest Sci. 2016;187:48-52.

18. Valle MR, Mendez L, Valdez M, Redondo M, Espinosa CM, Vargas M, et al. Integrated control of Boophilus microplus ticks in Cuba based on vaccination with the anti-tick vaccine Gavac. Exp Appl Acarol. 2004;34(3-4):375-82.

19. Inokuma H, Kemp DH. Establishment of Boophilus microplus infected with Babesia bigemina by using in vitro tube feeding technique. J Vet Med Sci. 1998;60(4):509-12.

20. Lew-Tabor AE, Bruyeres AG, Zhang B, Rodriguez VM. Rhipicephalus (Boophilus) microplus tick in vitro feeding methods for functional (dsRNA) and vaccine candidate (antibody) screening. Ticks Tick Borne Dis. 2014; 5(5):500-10. 
21. Willadsen P, Kemp DH, McKenna RV. Bloodmeal ingestion and utilization as a component of host specificity in the tick, Boophilus microplus. Z Parasitenkd. 1984;70(3):415-20.

22. Kröber T, Guerin PM. An in vitro feeding assay to test acaricides for control of hard ticks. Pest Manag Sci. 2007;63(1):17-22.

23. Kröber T, Guerin PM. In vitro feeding assays for hard ticks. Trends Parasitol. 2007;23(9):445-9.

24. Kuhnert F, Diehl PA, Guerin PM. The life-cycle of the bont tick Amblyomma hebraeum in vitro. Int J Parasitol. 1995;25(8):887-96.

25. Musyoki JM, Osir EO, Kiara HK, Kokwaro ED. Comparative studies on the infectivity of Theileria parva in ticks fed in vitro and those fed on cattle. Exp Appl Acarol. 2004;32(1-2):51-67.

26. Bonnet $\mathrm{S}$, Jouglin M, Malandrin L, Becker C, Agoulon A, L'Hostis M, et al. Transstadial and transovarial persistence of Babesia divergens DNA in Ixodes ricinus ticks fed on infected blood in a new skin-feeding technique. Parasitol. 2006;134(2):197-207.

27. Doube BM, Kemp DH. The influence of temperature, relative humidity and host factors on the attachment and survival of Boophilus microplus (Canestrini) larvae to skin slices. Int J Parasitol. 1979;9(5):449-54.

28. Kemp DH, Koudstaal D, Roberts JA, Kerr JD. Feeding of Boophilus microplus larvae on a partially defined medium through thin slices of cattle skin. Parasitol. 1975:70(APR):243.

29. Pierce AE, Pierce MH. A note on the cultivation of Boophilus microplus (Canestrini, 1887) (Ixodidae : Acarina) on the embryonated hen egg. Aust Vet J. 1956;32(6):144-6.

30. Waladde SM, Kemp DH, Rice MJ. Feeding electrograms and fluid uptake measurements of cattle tick Boophilus microplus attached on artificial membranes. Int J Parasitol. 1979;9(2):89-95.

31. Estrada-Pena A, Venzal JM, Nava S, Mangold A, Guglielmone AA. Labruna MBet al. Reinstatement of Rhipicephalus (Boophilus) australis (Acari: Ixodidae) with redescription of the adult and larval stages. J Med Entomol. 2012; 49(4):794-802

32. Abdel-Shafy S, Habeeb SM, El Namaky AH, Abou-Zeina HA. Scanning electron microscopy of nymphal and larval stages of the cattle tick Rhipicephalus (Boophilus) annulatus (Say) 1821 (Acari: Ixodidae). Glob Vet. 2013;10(1):01-8.

33. Tatchell RJ, Moorhouse DE. The feeding processes of the cattle tick Boophilus microplus (Canestrini). Parasitol. 1968;58(02):441-59.

34. Seifert GW, Springell PH, Tatchell RJ. Radioactive studies on the feeding of larvae, nymphs, and adults of the cattle tick, Boophilus microplus (Canestrini). Parasitol. 1968;58(02):415-30.

35. Bastos RG, Ueti MW, Knowles DP, Scoles GA. The Rhipicephalus (Boophilus) microplus Bm86 gene plays a critical role in the fitness of ticks fed on cattle during acute Babesia bovis infection. Parasit Vectors. 2010;3:111.

\section{Submit your next manuscript to BioMed Central and we will help you at every step:}

- We accept pre-submission inquiries

- Our selector tool helps you to find the most relevant journal

- We provide round the clock customer support

- Convenient online submission

- Thorough peer review

- Inclusion in PubMed and all major indexing services

- Maximum visibility for your research

Submit your manuscript at www.biomedcentral.com/submit 